\title{
Prevalence of ESBL Producing Escherichia coli and Klebsiella Species among Clinical Isolates and their in vitro Antimicrobial Susceptibility Pattern in a Tertiary Care Hospital
}

\author{
Sanjo Gupta, Veena Maheshwari* and Rajesh Shah \\ Department of Microbiology, RKDFMCH \& RC, Bhopal, India \\ *Corresponding author
}

\begin{abstract}
A B S T R A C T
E. coli and Klebsiella spp. are the main opportunistic pathogens under family Enterobacteriaceae. Cephalosporins are the first line of drugs against these organisms, but the inappropriate use of beta-lactam antibiotics render these organisms more vulnerable for the production of enzymes. Prevalence of ESBLs has resulted in widespread development

\section{Keywords}

ESBL, E.coli, Klebsiella spp., Ceftazidime, Ceftazidime+ clavulanic acid, Multidrug resistance.

\section{Article Info}

Accepted:

21 September 2017

Available Online:

10 October 2017 of drug resistance. The present study was done to detect the prevalence of ESBLs producing E. coli and Klebsiella spp with their antibiotic resistance patterns at TMMC Tertiary care hospital. A total no of 200 isolates were obtained from clinical samples from March to February 2015. Samples were inoculated on MacConkey and sheep blood agar. The organisms were identified based on standard microbiological techniques. Antimicrobial susceptibility testing was performed by Kirby-Bauer disc diffusion method, as per CLSI guidelines. Following screening with Cefotaxime (30 Â $\mu \mathrm{g}$ ) disc, resistance was then confirmed for ESBL production by phenotypic confirmatory disc diffusion test

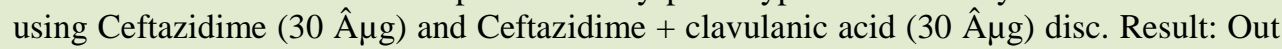
of 120 Escherichia coli isolates 80 isolates of Klebsiella species, 31(25.83\%) and 19 (23.75\%) were ESBL producers respectively. These ESBL producers showed high-degree of resistance to ampicillin (100\%) followed by ceftazidime, pipracillin and cefotaxime for both E. coli and Klebsiella species. The most sensitive antibiotics were found to be imipenem followed by Amikacin and meropenem. The present study highlights a relatively higher prevalence ESBL among E. coli and Klebsiella spp In view of these findings, we recommend that ESBL testing should be done routinely to reduce resistance to antibiotics and to strengthen the enforcement of infection control measures. It is pertinent for the treating clinicians so that they can make an informed choice as far as appropriate antibiotics and precautions are concerned.
\end{abstract}

\section{Introduction}

Beta-lactamases act as a major defense of Gram negative bacilli (GNB) against betalactam antibiotics. Treatment of Extended Spectrum Beta-Lactamase (ESBLs) producing Gram negative bacilli has posed a major challenge in hospitalized patients. ESBLs are $\beta$-lactamases conferring resistance to
oxyimino-Cephalosporins $\quad\left(3^{\text {rd }}\right.$ Generation Cephalosporins) and Aztreonam but not Cephamycins or Carbapenems.

Their numbers have increased significantly since 1989 and represent one of the largest groups of novel enzymes ${ }^{1}$. 
Ever since its discovery in 1983, pathogens producing Extended-spectrum- $\beta$ lactamases (ESBLs) have become an increasing cause of clinical concern for the clinicians for several reasons $^{2}$. First, systemic infections due to ESBL producing pathogens are associated with adverse clinical outcomes. Second, initially ESBLs producing pathogens was restricted to certain geographical areas which subsequently spread across the globe and their prevalence varies throughout the world. Third, primarily ESBLs was characterized among certain members of family Enterobacteriaceae such as E. coli and Klebsiella spp but then it spread among the other genera such as Citrobacter, Enterobacter and Proteus Spp. Finally, ESBL production is associated with more complex phenotypes due to multiple enzyme production which include: inhibitor-resistant ESBL variants, plasmid-borne AmpC, production of ESBLs in AmpC beta lactamases - producing bacteria, production of ESBLs in KPC (Carbapenamases)producing bacteria, enzyme hyperproduction and porin loss ${ }^{3}$.

Genus Klebsiella and Escherichia coli among family Enterobacteriaceae are frequently encountered opportunistic pathogens in clinical settings. Infection due to ESBL producers range from uncomplicated Urinary tract infection to life threatening sepsis. Cephalosporins are considered as first line drugs against Escherichia coli and Klebsiella species. The mechanism behind Cephalosporin resistant Klebsiella pneumoniae was the production of Extended Spectrum $\beta$-Lactamase (ESBLs).

In India, the prevalence of ESBL production varies from $10 \%$ to $84 \%$. ESBLs are more prevalent in Klebsiella spp. (pneumonia and oxytoca) followed by Escherichia coli ${ }^{4}$. With the spread of ESBL producing Gram negative bacilli (GNB) in hospitals worldwide, the aim of the present study was to find out the prevalence of ESBL producing Klebsiella species and Escherichia coli among clinical isolates and their antibiotic susceptibility patterns.

\section{Materials and Methods}

The present prospective study was carried out from March 2014 to February 2015 in the Department of Microbiology, in Teerthankar Mahaveer Medical College and Research Center Moradabad. During this period, a total of 200 isolates were obtained after processing of the clinical samples.

\section{Sample processing}

All clinical samples were inoculated on Blood agar, MacConkey's agar and the Klebsiella Species and Escherichia coli isolates that were identified based on colony morphology and biochemical reactions as per standard microbiological procedures ${ }^{5}$ were only considered for the study.

\section{Antimicrobial susceptibility testing}

The isolates were tested for antimicrobial susceptibility by modified Kirby-Bauer disk diffusion method on Muller Hinton agar plate as per clinical and laboratory standards institute guidelines (CLSI 2017) ${ }^{6}$.

The antibiotic discs used were Amikacin (30 $\mu \mathrm{g})$, gentamycin $(10 \mu \mathrm{g})$, ampicillin $(10 \mu \mathrm{g})$, ampicillin/sulbactam $(10 / 10 \mu \mathrm{g})$, ciprofloxacin(5 $\mu \mathrm{g})$, levofloxacin $(5 \mu \mathrm{g})$, chloramphenicol $(30 \mu \mathrm{g})$, co-trimoxazole $(1.25 / 23.75 \mu \mathrm{g})$, ceftriaxone $(30 \mu \mathrm{g})$, cefotaxime $(30 \mu \mathrm{g})$, ceftazidime $(30 \mu \mathrm{g})$, cefoxitin $(30 \mu \mathrm{g})$, amoxicillin clavulanic acid $(20 / 10 \mu \mathrm{g})$, tigecycline $(30 \mu \mathrm{g})$ piperacillintazobactum $(100 / 10 \mu \mathrm{g})$, imipenem $(10 \mu \mathrm{g})$ and meropenem $(10 \mu \mathrm{g})$, ertapenem $(10 \mu \mathrm{g})$. Norfloxacin $(10 \mu \mathrm{g})$ and nitrofurantoin (300 
$\mu \mathrm{g})$ were tested against isolates from urine samples only. Dehydrated media and antibiotic discs were obtained from Hi-Media, Mumbai.

Klebsiella pneumoniae ATCC 700603 and Esherichia coli ATCC 25922 strains were used as controls during this study.

\section{Screening for ESBL Producers for} Escherichia and Klebsiella species

According to CLSI guidelines, strains showing zone of inhibition less than $27 \mathrm{~mm}$ for Cefotaxime and less than $22 \mathrm{~mm}$ for Ceftazidime were taken as indicators for possible ESBL production. Phenotypic confirmatory Disc Diffusion test was used for confirmation amongst the potential ESBLproducing isolates. The discs of Ceftazidime $(30 \mathrm{mcg})$ and Ceftazidime plus Clavulanic + $(30 / 10 \mathrm{mcg})$ were placed on Mueller Hinton agar plates within a distance of $20 \mathrm{~mm}$ between two discs. Overnight incubation was done at $37^{\circ} \mathrm{C}$. An increase of zone diameter > $5 \mathrm{~mm}$ of Ceftazidime plus clavulanic acid in comparison to the zone diameter of Ceftazidime alone confirmed for ESBL production.

\section{Results and Discussion}

A total of 120 isolates of $E$. coli and 80 isolates of Klebsiella species isolated in the bacteriology laboratory of Department of Microbiology of Teerthankar Mahaveer Medical College and Research Center Moradabad were taken up during the study.

Among the 120 isolates of E. coli, 31 were identified as ESBL producers by phenotypic confirmatory disc diffusion test (PCDDT). Ceftazidime Clavulanic acid (93.54\%), Imipenem (83.87\%) and Meropenem $(80.64 \%)$ were the most effective antibiotics against ESBL producing E. coli. Imipenem
(88.76\%), Meropenem (86.51\%) and Ceftazidime Clavulanic acid (86.51\%) were the most effective antibiotics against nonESBL producing E. coli (Table 2).

Among the 80 isolates of Klebsiella, 19 were identified as ESBL producers by phenotypic confirmatory disc diffusion test (PCDDT). Imipenem (89.47\%), Meropenem (84.21\%) and Ceftazidime Clavulanic acid (84.21\%) were the most effective antibiotics against ESBL producing Klebsiella.

Imipenem (93.44\%), Meropenem (88.52\%), and Ceftazidime Clavulanic acid (85.24\%) were the most effective antibiotics against non-ESBL producing Klebsiella (Table 3).

In the present study, the percentage of ESBL producers among E. coli isolates was $25.83 \%$ and Klebsiella isolates was $23.75 \%$ (Table 4).

For the E. coli isolates, the most effective antibiotics were Imipenem $(75.83 \%)$ followed by Amikacin (74.16\%) and Meropenem (73.33). Highest resistance was seen against Ampicillin (80.83\%), Cotrimoxazole $(59.16 \%)$ and Ceftazidime $(57.5 \%)$ (Table 1 , Figure 1).

For the Klebsiella isolates, the most effective antibiotics were Imipenem $(81.25 \%)$ followed by Meropenem $(78.75 \%)$ and Ceftazidime Clavulanic acid $(78.75 \%)$.

Highest resistance was seen against Ampicillin (87.5\%), Cotrimoxazole (60.0\%) and cefepime $(56.25 \%)$ (Table 1, Figure 2).

In the present study, the prevalence of ESBL producing $E$. coli is $25.83 \%$ (31 ESBL producers out of total $120 \mathrm{E}$. coli isolates and the prevalence of ESBL producing Klebsiella spp. is $23.75 \%$ (19 ESBL producers out of total 80 Klebsiella spp. Isolates. The studies which are listed in Table 5 conducted in wide 
geographical areas of India by various authors, from the past 12 years reported a widely varied prevalence ranging from $10.10 \%$ to $87 \% .^{7-19}$

Major risk factors for colonization or infection with ESBL producing organisms are long term antibiotic exposure, prolonged ICU stay, nursing home residency, severe illness, residence in an institution with high rates of Ceftazidime and other 3-GC use and instrumentation or catherization. ${ }^{4}$ Though ESBLs are quite prevalent in Indian hospitals and community, they are not routinely tested by diagnostic Microbiology laboratories.

The possible factor could be different geographical locations, different levels of proficiency of Microbiology trained technical staffs, different antibiotics cut offs used, different guidelines followed and varied techniques used for E detection of ESBLs.

Table.1 Comparative studies in different regions of India

\begin{tabular}{|l|l|l|l|}
\hline Author & Place & Year & Prevalence (\%) \\
\hline Kumar et al., (7) & Hyderabad & 2006 & $10.10 \%$ \\
\hline Agrawal et al., (8) & Pune & 2008 & $16 \%$ \\
\hline Vemula et al., (9) & Kadappa & 2011 & $17 \%$ \\
\hline Variaya et al., (10) & Mumbai & 2008 & $20 \%$ \\
\hline Tankhiwale et al., (11) & Nagpur & 2004 & $25 \%$ \\
\hline Shukala et al., (12) & Aligarh & 2004 & $30.18 \%$ \\
\hline Babypadmini et a (13) & Coimbator & 2004 & $40 \%$ \\
\hline Metri et al., (14) & Bijapur & 2012 & $44.90 \%$ \\
\hline Taneja et al., (15) & Chandigarh & 2008 & $51.20 \%$ \\
\hline Hawser et al., SMART study (16) & 7 hospitals India & 2010 & $55 \%$ \\
\hline Rao et al., (17) & Davangere & 2008 & $62.20 \%$ \\
\hline Rudresh et al., (18) & Bangalore & 2011 & $63.30 \%$ \\
\hline Sharma et al., (19) & Jaipur & 2013 & $67.04 \%$ \\
\hline
\end{tabular}

Table.2 Antibiotic sensitivity patterns of E. coli and Klebsiella species

\begin{tabular}{|l|l|l|l|l|}
\hline \multirow{2}{*}{ Antibiotics } & E. coli $\mathrm{n}=(45)$ & \multicolumn{2}{l|}{ Klebsiella $\mathrm{n}=(15)$} \\
\cline { 2 - 5 } & $\mathrm{S}(\mathrm{n} \%)$ & $\mathrm{R}(\mathrm{n} \%)$ & $\mathrm{S}(\mathrm{n} \%)$ & $\mathrm{R}(\mathrm{n} \%)$ \\
\hline Amikacin & $89(74.16)$ & $31(25.83)$ & $62(77.5)$ & $18(22.5)$ \\
\hline Ampicillin & $23(19.16)$ & $97(80.83)$ & $10(8.33)$ & $70(87.5)$ \\
\hline Ceftazidime & $51(42.5)$ & $69(57.5)$ & $36(45)$ & $44(55)$ \\
\hline Ceftazidime +Clavulanic acid & $77(64.16)$ & $43(35.83)$ & $63(78.75)$ & $17(21.25)$ \\
\hline Cefepime & $52(43.33)$ & $68(56.66)$ & $35(43.75)$ & $45(56.25)$ \\
\hline Ciprofloxacin & $71(54.16)$ & $49(40.83)$ & $50(62.5)$ & $30(37.5)$ \\
\hline Levofloxacin & $73(60.83)$ & $47(39.16)$ & $49(61.25)$ & $31(38.75)$ \\
\hline Pipracillin & $56(46.66)$ & $64(53.33)$ & $39(48.75)$ & $41951.25)$ \\
\hline Pipracillin+Tazobactum & $84(70)$ & $26(21.66)$ & $36(30.66)$ & $18(22.5)$ \\
\hline Gentamicin & $78(65.33)$ & $42(35)$ & $55(68.75)$ & $25(31.25)$ \\
\hline Co-trimoxazole & $49(40.83)$ & $71(59.16)$ & $32(40)$ & $48(60)$ \\
\hline Nitrofruntine & $82(68.33)$ & $38(31.66)$ & $54(67.5)$ & $26(32.5)$ \\
\hline Imipenem & $91(75.83)$ & $29(24.16)$ & $65(81.25)$ & $15(18.75)$ \\
\hline Meropenem & $88(73.33)$ & $32(26.66)$ & $3(78.75)$ & $17(21.25)$ \\
\hline
\end{tabular}


Table.3 Antibiotic sensitivity pattern of ESBL and non-ESBL producing E. coli

\begin{tabular}{|l|l|l|l|l|}
\hline E. coli $\mathrm{n}=(120)$ & \multicolumn{2}{|l|}{ ESBL producers $(31)$} & \multicolumn{2}{l|}{ Non-ESBL producers $(89)$} \\
\hline Antibiotics & $\mathrm{S}(\mathrm{n} \%)$ & $\mathrm{R}(\mathrm{n} \%)$ & $\mathrm{S}(\mathrm{n} \%)$ & $\mathrm{R}(\mathrm{n} \%)$ \\
\hline Amikacin & $19(61.29)$ & $12(38.70)$ & $59(66.29)$ & $30(34.88)$ \\
\hline Ampicillin & 00 & $31(100)$ & $23(25.84)$ & $56(49.43)$ \\
\hline Ceftazidime & $6(19.35)$ & $25(80.64)$ & $45(50.6)$ & $44(50.56)$ \\
\hline Ceftazidime + Clavulanic acid & $29(93.54)$ & $2(6.45)$ & $77(86.51)$ & $12(86.51)$ \\
\hline Cefepime & $52(22.58)$ & $24(77.41)$ & $43(48.31)$ & $46(51.68)$ \\
\hline Ciprofloxacin & $15(48.38)$ & $16(51.61)$ & $28(31.46)$ & $61(68.53)$ \\
\hline Levofloxacin & $17(54.83)$ & $14(45.16)$ & $34(38.20)$ & $55(61.79)$ \\
\hline Pipracillin & $10(32.25)$ & $21(67.74)$ & $56(62.92)$ & $33(62.92)$ \\
\hline Pipracillin+Tazobactum & $15(48.38)$ & $16(51.61)$ & $61(68.53)$ & $28(31.64)$ \\
\hline Gentamicin & $13(41.93)$ & $18(58.06)$ & $53(59.55)$ & $36(40.44)$ \\
\hline Co-trimoxazole & $9(29.03)$ & $22(70.96)$ & $29(32.58)$ & $66(74.15)$ \\
\hline Nitrofruntine & $14(45.16)$ & $17(54.83)$ & $43(48.31)$ & $46(51.68)$ \\
\hline Imipenem & $2683.87)$ & $5(16.12)$ & $79(88.76)$ & $10(11.23)$ \\
\hline Meropenem & $25(80.64)$ & $6(19.35)$ & $77(86.51)$ & $12(13.43)$ \\
\hline
\end{tabular}

Table.4 Antibiotic sensitivity pattern of ESBL and non-ESBL producing Klebsiella

\begin{tabular}{|l|l|l|l|l|}
\hline Klebsiella $\mathrm{n}=(80)$ & \multicolumn{2}{|l|}{ ESBL producers $(19)$} & \multicolumn{2}{l|}{ Non-ESBL producers $(61)$} \\
\hline Antibiotics & $\mathrm{S}(\mathrm{n} \%)$ & $\mathrm{R}(\mathrm{n} \%)$ & $\mathrm{S}(\mathrm{n} \%)$ & $\mathrm{R}(\mathrm{n} \%)$ \\
\hline Amikacin & $9(47.36)$ & $10(52.63)$ & $33(54.09)$ & $28(45.90)$ \\
\hline Ampicillin & 00 & $19(100)$ & $24(39.34)$ & $27(44.26)$ \\
\hline Ceftazidime & $4(21.05)$ & $15(78.94)$ & $28(45.90)$ & $33(54.09)$ \\
\hline Ceftazidime ${ }_{+}$Clavulanic acid & $16(84.21)$ & $3(15.78)$ & $52(85.24)$ & $9(14.75)$ \\
\hline Cefepime & $5(26.31)$ & $14(73.68)$ & $31(50.81)$ & $30(49.18)$ \\
\hline Ciprofloxacin & $8(42.10)$ & $11(57.89)$ & $29(47.54)$ & $32(52.45)$ \\
\hline Levofloxacin & $9(47.36)$ & $10(52.63)$ & $32(52.45)$ & $29(47.54)$ \\
\hline Pipracillin & $7(36.84)$ & $12(63.15)$ & $27(44.26)$ & $34(55.73)$ \\
\hline Pipracillin+Tazobactum & $11(57.89)$ & $8(42.10)$ & $37(60.65)$ & $24(39.34)$ \\
\hline Gentamicin & $9(47.36)$ & $10(52.63)$ & $29(47.54)$ & $32(52.45)$ \\
\hline Co-trimoxazole & $7(36.84)$ & $12(63.15)$ & $22(36.06)$ & $39(63.93)$ \\
\hline Nitrofruntine & $10(52.63)$ & $9(47.36)$ & $33(54.09)$ & $28(45.90)$ \\
\hline Imipenem & $17(89.47)$ & $2(10.52)$ & $57(93.44)$ & $4(6.55)$ \\
\hline Meropenem & $16(84.21)$ & $3(15.78)$ & $54(88.54)$ & $7(11.47)$ \\
\hline
\end{tabular}

Table.5 Percentage of ESBL and non-ESBL producing Klebsiella and Escherichia coli

\begin{tabular}{|l|l|l|l|}
\hline Total samples & 200 & ESBL producers $(50=25 \%)$ & Non-ESBL producers $(150=75 \%)$ \\
\hline Klebsiella Species & $80=40 \%$ & $19=23.75 \%$ & $61=76.25 \%$ \\
\hline Escherichia coli & $120=60 \%$ & $31=25.83 \%$ & $89=74.16 \%$ \\
\hline
\end{tabular}


Fig.1 Antibiotic sensitivity pattern of Escherichia coli

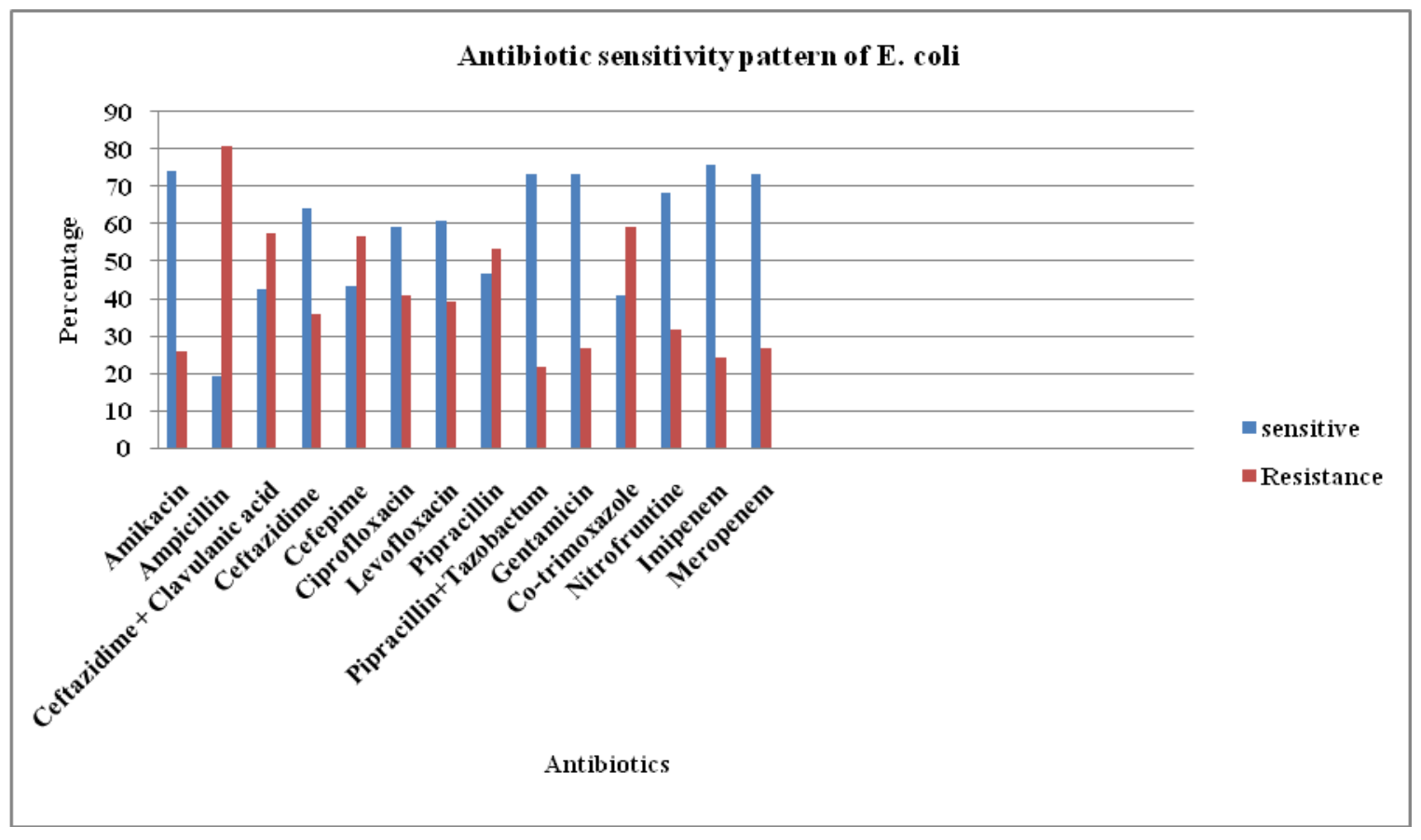

Fig.2 Antibiotic sensitivity pattern of Klebsiella species

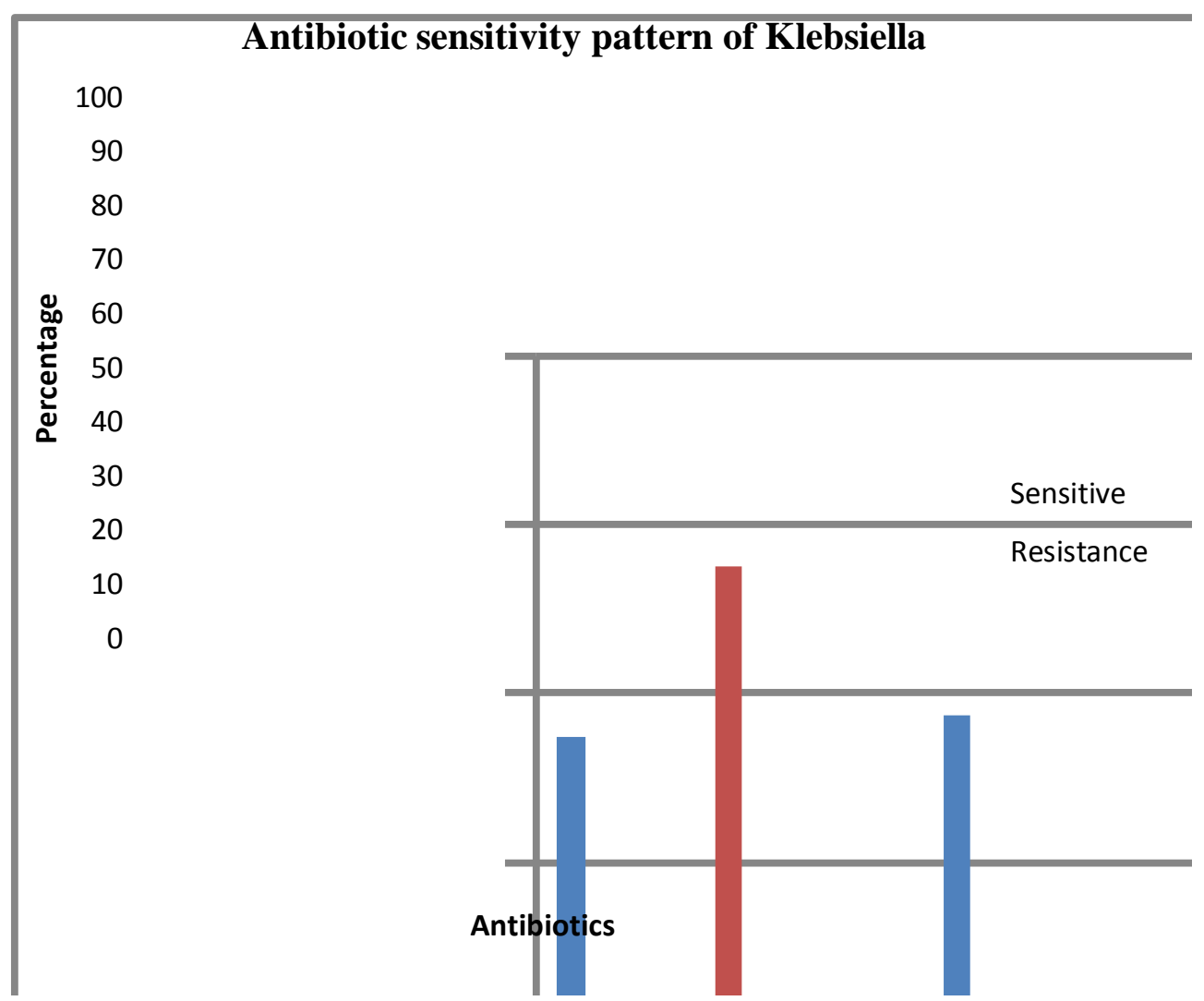


In developing country like India, ESBL detection and its antimicrobial susceptibility testing should be routinely undertaken to enable the clinician to select appropriate drug regimen

Klebsiella species and Escherichia coli remain the major ESBL producing organisms isolated worldwide which are recommended to be routinely tested and reported by the Clinical and Laboratory Standards Institute. ${ }^{6}$

ESBLs are more prevalent in Klebsiella spp. (pneumonia and oxytoca) followed by Escherichia coli ${ }^{4}$. In the present study, Escherichia coli $(60 \%)$ was the most frequent and statistically significant ESBL producing organisms $(\mathrm{p}<0.05)$ followed by Klebsiella spp. (40\%). Predominant prevalence of ESBL among Escherichia coli isolates was also reported in a study by Ananthakrishnan et al., ${ }^{20}$ and Kumar et al., ${ }^{21}$. A similar study from central India reported the incidence of $69 \%$ and $41 \%$ ESBL producing isolates of E. coli and Klebsiella pneumoniae respectively. ${ }^{22}$

In the present study, ESBL detection was carried out by the Phenotypic Confirmatory Disc Diffusion test (PCDDT) on 120 isolates of E. coli and 80 isolates of Klebsiella species. Imipenem was found to be the most effective $(75.83 \%)$ antibiotic followed by Amikacin (74.16\%) and Meropenem (73.33\%). Our findings correlate with a similar study done by Chika et al., ${ }^{23}$ and Sharma et al., ${ }^{24}$ where imipenem sensitivity was reported to be $84.6 \%$ and $96.2 \%$ among the isolates. The isolates of $\mathrm{E}$. coli in our study were found to be most resistance to Ampicillin, Cotrimoxazole and Ceftazidime which coincides well with studies done by Chika et al., and Sharma et al., and Sarojamma et al., ${ }^{25}$

Amongst the Klebsiella isolates, Imipenem was found to be the most effective $(81.25 \%)$ followed by Amikacin (77.5\%) and Piperacillin + Tazobactum (77.5\%). These findings correlate with a study done by Chander et al., in which $100 \%$ sensitivity of Klebsiella to Imipenem was reported. However, resistance to Amikacin and Piperacillin + Tazobactum was only $22.5 \%$.In our study, 31 isolates $(25.83 \%)$ of $E$. coli and 19 isolates $(23.75 \%)$ of Klebsiella spp. were found to be ESBL producers.

Ceftazidime + Clavulanic acid(93.5\%) was the most effective antibiotic against ESBL producing $E$. coli followed by Imipenem (83.87\%) and Meropenem (80.64\%). Against ESBL producing Klebsiella, Imipenem was most effective $(89.47 \%)$ followed by Ceftazidime + Clavulanic acid $(84.21 \%)$ and Piperacillin + Tazobactum (84.21\%). These findings are marginally better than studies conducted by Chika et al., 7 where ESBL producing Klebsiella were 26\%. However, Chander et al., 10 had lower ESBL rates for E. coli (13.5\%) and Klebsiella (16.5\%).

In a study done by Mumtaz $\mathrm{S}$ et al., ${ }^{11}$, antibiotic sensitivity correlates with our study where most of the isolates are sensitive to Imipenem $(95.7 \%)$ followed by Amikacin $(76 \%)$.

As observed in our study, Imipenem was effective among both ESBL and non-ESBL producing E. coli and Klebsiella species.

It should be kept in mind that Carbapenems should only be used in emergency and life threatening situations and not as a part of routine use so that the therapeutic value of these drugs is preserved and available when absolutely necessary $^{12}$.

A well designed antibiotic policy, effective implementation of the same, awareness on ESBLs in hospitals are important points to be taken into account while handling resistant 
organisms such as ESBL producers.

The present study highlights a relatively higher prevalence ESBL among E. coli and Klebsiella spp In view of these findings, we recommend that ESBL testing should be done routinely to reduce resistance to antibiotics and to strengthen the enforcement of infection control measures. It is pertinent for the treating clinicians so that they can make an informed choice as far as appropriate antibiotics and precautions are concerned.

\section{References}

1. Agrawal, P., AN Ghosh, S Kumar, B Basu, K Arnold, R.S., Thom, K.A., Sharma, S., Phillips, M., Johnson, J.K. and Morgan, D.J. (2011). Emergence of Klebsiella pneumoniae Carbapenemase (KPC)-Producing Bacteria. South Med. J; 104(1): 4045.

2. Carvalhaes, C.G., Picao, R.C., Nicoletti, A.G., Xavier, D.E. and Gales, A.C. 2010. Cloverleaf test (modified Hodge test) for detecting carbapenemase production in Klebsiella pneumoniae: be aware of false positive results. J Antimicrob Chemother ; 65: 249-251.

3. Chauhan, K., Pandey, A., Asthana, A.K. and Madan, M. 2015. Evaluation of phenotypic tests for detection of Klebsiella pneumoniae carbapenemase and metallo-beta-lactamase in clinical isolates of Escherichia coli and Klebsiella species. Indian $J$ Pathol Microbiol ;58:31-5.

4. Clinical and Laboratory Standards Institute. Performance Standards for Antimicrobial Susceptibility Testing: Twenty First Informational Supplement M100-S21. CLSI, Wayne, PA: USA; 2011.

5. Datta, P., Gupta, V., Shivani, G. and Chander J. 2012. Phenotypic method for differentiation of carbapenemases in Enterobacteriaceae: Study from North
India. Indian $J$ Pathol Microbiol ;55(3):357-360.

6. Fattouh, M., El-din, A.N. and Omar, M.A. 2015. Detection of Klebsiella pneumoniae Carbapenemase (KPC) Producing Gram negative superbugs: An emerging cause of multidrug-resistant infections in general surgery department of Sohag University Hospital, Egypt. Int.J.Curr.Microbiol.App.Sci ; 4(5): 1-15

7. Girlich, D., Poirel, L., and Nordmann, P. 2012.Value of the modified Hodge test for detection of emerging carbapenemases in Enterobacteriaceae. $J$ Clin Microbiol;

50(2): 477479- 9

8. Girlich, D., Halimi, D., Zambardi, G. and Nordmann P.2013. Evaluation of Etest MBL strips for detection of KPC and metallo-carbapenemases

Enterobacteriaceae. Diagn Microbiol Infect Dis ; 77: 200-201.

9. Hrabak, J., Chudackova, E., and Papagiannitsis, C.C. 2014. Detection of carbapenemases in Enterobacteriaceae: a challenge for diagnostic microbiological laboratories. Clin Microbiol Infect ; 20: 839-853.

10. Miriagou, V., Tzelepi, E., Kotsakis, S.D., Daikos, G.L, Casal, J.B. and Tzouvelekis LS.2013. Combined disc methods for the detection of KPC- and/or VIM-positive Klebsiella pneumoniae: improving reliability for the double carbapenemase producers Clin Microbiol Infect ; 19: E412-E415 10.1111/14690691.

11. Nadkarni, A.S., Schliep ,T. and Khan, L. 2009. Cluster of bloodstream infections caused by KPC-2 Carbapenemaseproducing Klebsiella pneumoniae in Manhattan. Am J. Infect. Control; 37: 121- 126.

12. Nordmann, P., Poirel, L. and Dortet, L. 2012. Rapid detection of carbapenemase- 
producing Enterobacteriaceae. Emerg Infect Dis; 18: 1503-1507.

13. Patel, G., Huprikar, S and Factor, S.H. 2008. Outcomes of carbapenem-resistant Klebsiella pneumoniae infection and the impact of antimicrobial and adjunctive therapies. Infect. Control Hosp. Epidemiol.; 29: 1099-1106.

14. Queenan, A.M.and Bush, K. 2007. Carbapenemases: the versatile betalactamases. Clin Microbiol Rev; 20:440 e58

15. Schwaber, M.J. and Carmeli, Y. 2014.An ongoing national intervention to contain the spread of carbapenem-resistant Enterobacteriaceae. Clin Infect Dis; 58: 697-703.

16. Tsakris, A., Kristo, I. and Poulou ,A. 2009. Evaluation of boronic acid disk tests for

differentiating KPC-possessing Klebsiella pneumoniae isolates in the clinical laboratory. J Clin. Microbio; 47: 362367.
17. Tsakris, A., Poulou, A., Pournaras, S., Voulgari, E., Vrioni, G., ThemeliDigalak, K., Dimitra Petropoulou, D. and Sofianou D. 2010. A simple phenotypic method for the differentiation of metallo beta-lactamases and class A KPC carbapenemases in Enterobacteriaceae clinical isolates. J. Antimicrob.Chemother; 65: 1664- 1671.

18. Yong, D., Toleman, M.A., Giske, C.G., Cho, H.S., Sundman, K., Lee, K. and Walsh, T.R. 2009. Characterization of a new metallo-beta- lactamase gene, blaNDM-1, and a novel erythromycin esterase gene carried on a unique genetic structure in Sequence Type 14 from India. Antimicrob. Agents Chemother; 53: 5046- 5054

19. Wattal, C., Goel, N., Oberoi, J.K., Raveendran, R., Datta, S. and Prasad, K.J. 2010.Surveillance of multidrugresistant organisms in tertiary care hospital in Delhi, India. $J$ Assoc Physicians India ; 58:32-36.

\section{How to cite this article:}

Sanjo Gupta, Veena Maheshwari and Rajesh Shah. 2017. Prevalence of ESBL Producing Escherichia coli and Klebsiella species among clinical isolates and their invitro antimicrobial susceptibility pattern in a tertiary care hospital. Int.J.Curr.Microbiol.App.Sci. 6(10): 22952303. doi: https://doi.org/10.20546/ijcmas.2017.610.272 